\title{
A PHYSICAL MODEL FOR THE JOINT EVOLUTION OF QSOS AND SPHEROIDS
}

\author{
G.L. Granato, ${ }^{1}$ L. Silva, ${ }^{2}$ G. De Zotti, ${ }^{1}$ A. Bressan ${ }^{1}$ and L. Danese ${ }^{3}$ \\ ${ }^{1}$ INAF, Padova, Italy, ${ }^{2}$ INAF, Trieste, Italy, ${ }^{3}$ SISSA, Trieste, Italy
}

\section{The model}

We present a detailed, physically grounded, model for the early coevolution of spheroidal galaxies and of active nuclei at their centers. The model is based on very simple recipes, that can be easily implemented. The main components and the transfer processes accounted for are depicted in Fig. 1, while we defer to Granato et al. (2004) for a full description. In summary, we start from the diffuse gas within the dark matter halo falling down into the star forming regions at a rate ruled by the dynamic and the cooling times. Part of this gas condenses into stars, at a rate again controlled by the local dynamic and cooling times. But the gas also feels the feedback from supernovae and from active nuclei, heating it and possibly expelling it from the potential well. Also the radiation drag on the cold gas decreases its angular momentum, causing an inflow into a reservoir around the central black hole. Viscous drag then causes the gas to flow from the reservoir into the black hole, increasing its mass and powering the nuclear activity. Among the most novel point of our work with respect to other semi-analytic approaches, we point out the treatment of the evolution of QSO activity in the forming spheroids, and of the resulting effects on galaxy evolution.

\section{Results}

In the shallower potential wells (corresponding to lower halo masses and, for given mass, to lower virialization redshifts), the supernova heating is increasingly effective in slowing down the star formation and in driving gas outflows, resulting in an increase of star/dark-matter ratio with increasing halo mass. As a consequence, the star formation is faster within the most massive halos, and the more so if they virialize at substantial redshifts. Thus, in keeping with the proposition by Granato et 


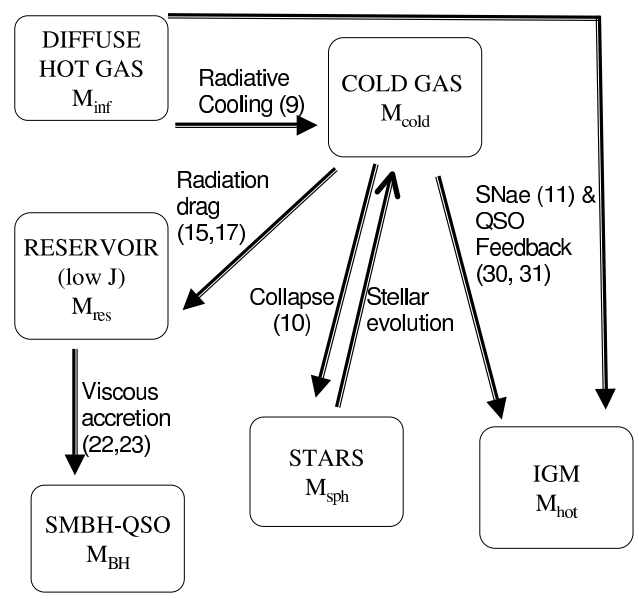

Figure 1. Scheme of the baryonic components included in the model (boxes), and of the corresponding mass transfer processes (arrows).

al. (2001), physical processes acting on baryons effectively reverse the order of formation of galaxies compared to that of dark-matter halos.

A higher star-formation rate also implies a higher radiation drag, resulting in a faster loss of angular momentum of the gas (Umemura 2001) and, consequently, in a faster inflow towards the central blackhole. In turn, the kinetic energy carried by outflows driven by active nuclei through line acceleration is proportional to $L_{\mathrm{QSO}}^{3 / 2}$, and this mechanism can inject in the interstellar medium a sufficient amount of energy to unbind it. The time required to sweep out the interstellar medium, thus halting both the star formation and the black-hole growth, is again shorter for larger halos. For the most massive galaxies $\left(M_{\mathrm{vir}} \gtrsim 10^{12} M_{\odot}\right)$ virializing at $3 \lesssim z_{\text {vir }} \lesssim 6$, this time is $<1 \mathrm{Gyr}$, so that the bulk of the star-formation may be completed before type Ia supernovae can significantly increase the $\mathrm{Fe}$ abundance of the interstellar medium; this process can then account for the $\alpha$-enhancement seen in the largest galaxies.

The interplay between star formation and nuclear activity determines the relationship between the black-hole mass and the mass, or velocity dispersion, of the host galaxy, as well as the black-hole mass function. As illustrated by Figs. 2 and 3, the model predictions are in excellent agreement with the observational data. A specific prediction of the model is a substantial steepening of the $M_{\mathrm{BH}^{-}} \sigma$ relation for $\sigma \lesssim 150 \mathrm{~km} \mathrm{~s}^{-1}$ : the mass of the $\mathrm{BH}$ associated to less massive halos is lower than expected from an extrapolation from higher masses, because of the combined ef- 


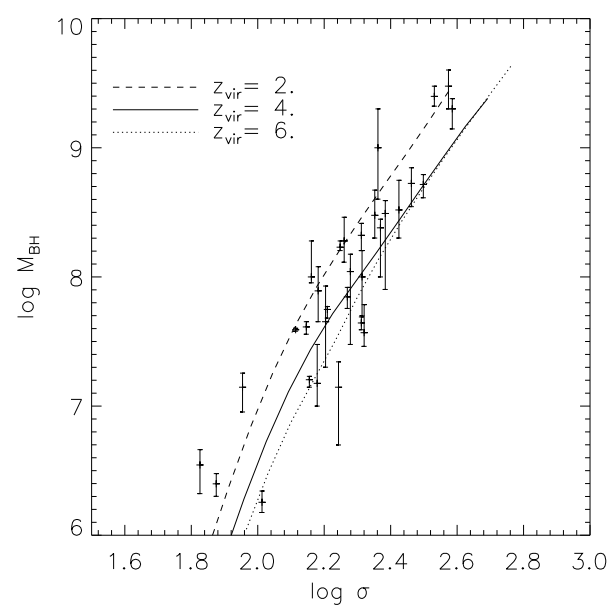

Figure 2. Predicted relationship between black-hole mass and line-of-sight velocity dispersion of the host galaxy for different virialization redshifts.

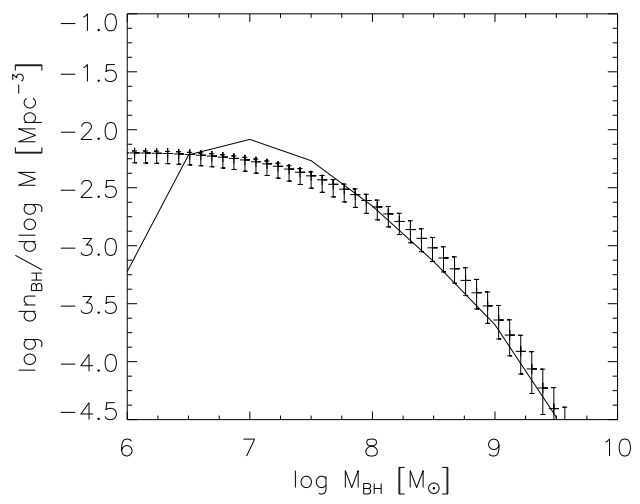

Figure 3. Predicted local black-hole mass function compared with the recent estimate by Shankar et al. (in preparation). The total mass density in BHs they derive is $\rho_{B H} \simeq 4 \times 10^{5} M_{\odot} \mathrm{Mpc}^{-3}, 25 \%$ less than our model. The decline of the model at low $M_{\mathrm{BH}}$ is due to having considered only objects with $M_{\mathrm{vir}} \geq 2.5 \times 10^{11} M \odot$.

fect of SN heating, which is increasingly effective with decreasing galaxy mass in hindering the gas inflow towards the central $\mathrm{BH}$, and of the decreased radiation drag.

The ratio between QSO and SNae feedback is an increasing function of the mass. At low mass the cumulative effect of QSO is almost negligible with respect to that of SNae, but it becomes dominant at intermedi- 
ate mass (typically by a factor of a few) and high mass (by a factor $\gtrsim 10)$. Note that the QSO effect usually increases exponentially with time, while that of SNae increases more slowly. Thus the instantaneous QSO effect becomes dominant, if ever, only a few e-folding times before the maximum of QSO activity.

Coupling the model with GRASIL (Silva et al. 1998), the code computing in a self-consistent way the chemical and spectrophotometric evolution of galaxies over a very wide wavelength interval, we have obtained predictions for the sub-mm counts and the corresponding redshift distributions as well as for the redshift distributions of sources detected by deep K-band surveys, which proved to be extremely challenging for all the current semi-analytic models. The results, shown by Fig. 4, are again very encouraging.

The cosmic history of $\mathrm{BH}$ accretion, is in keeping with the common notion that quasar activity peaks at redshift 2-3. Also, the formation rate of spheroids derived in this paper is consistent with that we computed in Granato et al. 2001 from a deconvolution of the high-z luminosity function of QSO.
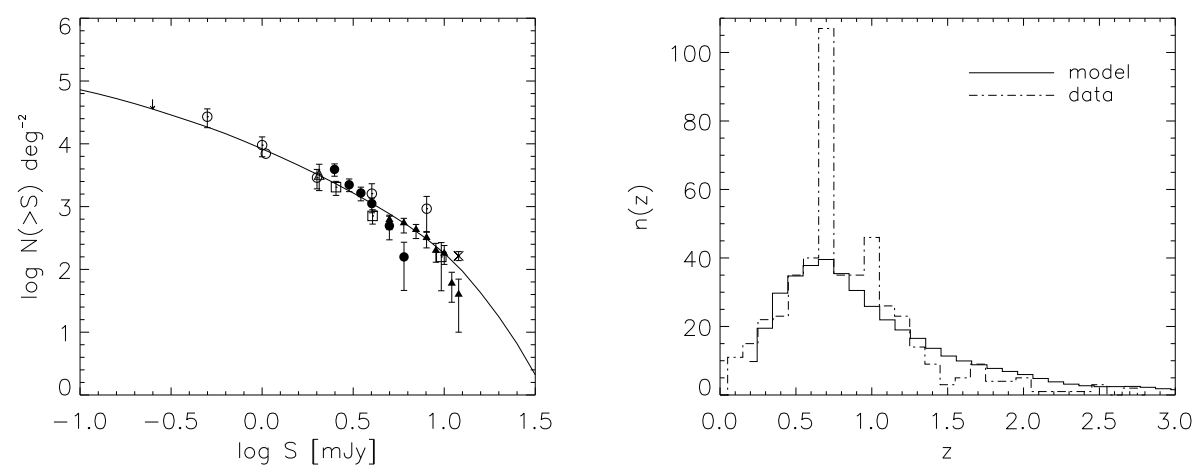

Figure 4. Left: predicted $850 \mu \mathrm{m}$ extragalactic counts compared with observed SCUBA counts. Right: predicted redshift distribution of galaxies brighter than $K=$ 20 compared with the results of the K20 survey

\section{References}

Granato, G.L., Silva, L., Monaco, P., Panuzzo, P., Salucci, P., De Zotti, G., \& Danese, L. 2001, MNRAS, 324, 757

Granato, G.L., De Zotti, G., Silva,L., Bressan, A. \& Danese, L. 2004, ApJ, in press

Silva, L., Granato, G.L., Bressan, A., \& Danese, L. 1998, ApJ, 509, 103

Umemura, M. 2001, ApJ, 560, L29 